\title{
Anti-PCSK9 Vaccines - An Emerging Therapeutic Innovative Game Changer in Dyslipidemia and Beyond
}

\author{
Mrinal Kanti Das ${ }^{1, ~ *, ~ T a p a n ~ S i n h a ~}{ }^{2}$, Mahesh Kumar Chaudhary ${ }^{1}$ \\ ${ }^{1}$ Department of Cardiology, C. K. Birla Hospitals, Kolkata, India \\ ${ }^{2}$ Department of Cardiology, Kothari Medical Centre, Kolkata, India
}

Email address:

drmkdas2001@yahoo.co.in (M. K. Das)

${ }^{*}$ Corresponding author

\section{To cite this article:}

Mrinal Kanti Das, Tapan Sinha, Mahesh Kumar Chaudhary. Anti-PCSK9 Vaccines - An Emerging Therapeutic Innovative Game Changer in Dyslipidemia and Beyond. American Journal of Internal Medicine. Special Issue: Dyslipidemia: Flash Back and Vision Ahead.

Vol. 8, No. 6, 2020, pp. 250-253. doi: 10.11648/j.ajim.20200806.11

Received: September 23, 2020; Accepted: October 15, 2020; Published: October 26, 2020

\begin{abstract}
Proprotein convertase subtilisin/kexin type 9 serine protease (PCSK9) regulates the cholesterol metabolism by genetic mechanism mediating the LDL receptor degradation. Gain of the functions of the PCSK9 variants resulted in high level of LDL-cholesterol and loss of function in low level of the LDL-cholesterol level. The low level of LDL-cholesterol was associated with significant decrease in coronary artery disease. The genetic insight of lipid homeostasis and complex molecular biochemistry triggered a domain of active pharmacological research with prospect of introducing paradigm shift in primary and secondary prevention of atherosclerotic cardiovascular disease (ASCVD) in group of non-responders to anti-cholesterol treatment. The result has been the discovery of the PCSK9 inhibitors and vaccines. The former got the statutory approval by Federal Drug Administration (FDA) in conditions namely patients with heterozygous familial hypercholesterolemia (FH), individuals with ASCVD requiring further lowering of LDL-cholesterol levels. This has also important role in statin-intolerant patients. The various international guidelines have put the concept of the therapeutic approach in acute coronary syndrome (ACS) specially in high risk post-MI patients. However the present article will focus more on the PCSK9 vaccine and the encouraging evidences of its pleotropic effects in treatment of chronic clinical conditions and cancer. There are few challenges which need to be further explored especially considering the cost-benefit ratio. There is also the need of further research focusing on the expansion of the indications.
\end{abstract}

Keywords: Proprotein Convertase Subtilisin/Kexin Type 9 Serine Protease (PCSK9), PCSK9 Inhibitors, Dyslipidemia and Beyond, Pleotropic Actions

\section{Introduction}

Reduction of Low Density Lipoprotein (LDL) has been associated with primary and secondary prevention of Cardiovascular (CVD) morbidity and mortality in large number of trials [1]. Since 4S Trial (1994) showed 29\% secondary reduction in mortality over 5 years, emphasis has eminently shifted to therapeutic virtues of statins by lowering LDL. Meta-analysis of 11 trials on statins involving 65,229 patients with high risk of CVD showed 9\% median risk reduction in mortality with $95 \% \mathrm{CI}$ in primary prevention [2]. However, only $0.55 \%$ absolute increase in survival was noted by statin in low risk patients [3]. On statistical terms, only two primary prevention studies with considerably higher LDL, JUPITER and WOSCOPS produced marginally significant $(P=0.02 \&$ 0.04 respectively) survival benefit. On the contrary, both $4 \mathrm{~S}$ trial and lipid trials dealing with secondary prevention showed only 3\% increased overall survival over 5-6 years in absolute terms. In largest secondary prevention Heart Protection Study (HPS) long term benefit was absent after 11 years [4]. On the other hand, despite optimum lifestyle modifications and therapeutic interventions about $33 \%$ patients fail to show more than $15 \%$ reduction of LDL level in 10 years. Using propensity-score matching over 5-year cumulative incidences of Major Adverse Cardiac Events (MACE) did not show any statistical difference between normal responders and non-responders to cholesterol 
lowering treatment [5]. Meta-analysis of Cholesterol Treatment Trialists' Collaborators (CTTC) included data from $26 \mathrm{RCTs}$ on statins involving $>170,000$ participants. It was observed that $39 \mathrm{mg} / \mathrm{dl}$ absolute reduction of LDL was associated with $10 \%$ reduction in all-cause mortality over 5 years. Thus, while association of LDL with increased morbidity and mortality from CVD is established, promise of substantial therapeutic efficacy of statins in reduction of absolute number of mortality has remained elusive. This scientific dysphoria lead to new metabolic exposition of LDL and its association with CVD. In this not so encouraging scenario, discovery of Proprotein Convertase Subtilisin / Kexin 9 (PCSK9) in 2003 and its inhibition has introduced scopes of paradigm shift in approach to the management of cholesterol homeostasis.

In this article we first intend to briefly explore role of PCSK9 enzyme encoded in chromosome 1 in LDL metabolism. Then we shall tread on the road which will lead us to the point of two therapeutic avenues of attenuation of PCSK9 by inhibitors and vaccination, with our emphasis given on vaccines. Therapeutic actions are described with implication on CVD. Our discussion will also include pleotropic effects of PCSK9 and extensions of its use in treatment of cancer. In the concluding section, despite insertion of caveats, we shall try to place PCSK9 inhibition through vaccination as most important breakthrough in therapeutic innovation of pharmacological prevention of CVD since advent of statins antagonizing HMG CoA-R.

At the very onset, we must emphasize that this article would primarily remain oriented at clinical aspect of antiPCSK9 vaccine and will skip over complex issues of molecular biology but for very cursory mentions.

\section{Biological Mechanism of PCSK9}

In pre PCSK9 era cholesterol homeostasis was considered to be strictly intracellular. Now, under impact of PCSK9, metabolism is found to be both intra and extra cellular, predominantly involving hepatocyte LDL receptors (LDLr), but are also expressed in small intestine and kidney. LDLr has life span of about 20 hours and recycles every 10 minutes [6]. PCSK9 down regulate LDLr, diminishing clearance of LDL from circulation and thus increasing propensity for CVD [7]. At any point of time, about $50 \%$ of plasma PCSK9 molecule is associated with LDL in 1:500-1000 ratio. In heterozygotic and homozygotic autosomal dominant familial hypercholesterolemia ( $\mathrm{HeFH} \& \mathrm{HoFH})$, non-responders to anti-cholesterol therapy, PCSK9 enzyme is associated with enhanced activity (gain-of-function) resulting in enhanced degradation of LDLr and increase in plasma LDL through reduced internalization. On the other hand loss-of-function mutation and induced inhibition of PCSK9 is associated with increase in LDLr expression and reduction of LDL level through internalization. Interestingly, in both $\mathrm{HeFH}$ and HOFH, statins enhance, i.e. up-regulate both LDLr and PCSK9 causing statin paradox: i.e. simultaneous beneficial increase in LDLr and damaging increase of PCSK9 [8]. Steerol-Regulatory Element (SRE) up-regulate PCSK9 which in turn impairs actions of statins and ezetimibe in lowering LDL.

Under this backdrop, novel approach for reduction of LDL through antagonizing actions of PCSK9 on LDLr is designed using monoclonal antibodies (mAbs- Alirocumab, svolocumab), anti-PCSK9 vaccines (small interfering RNAs [siRNA] and virus-like particle [VLP] peptides), antisense oligonucleotides (ASO), adenectin mimetic peptides, and several other vaccination strategies. Out of this prodigious array of choices, mAbs and vaccinations against PCSK9 are likely to make impact in clinical practice.

\subsection{Road to Anti-PCSK9 Modulation Treatment}

In the history of medical therapeutics there had not been very many incidences of invention of treatment modalities following close in heel of development of its basic science as in case of PCSK9 inhibition.

\subsection{Timeline of Emergence of Therapeutic Utility of PSCK9 Inhibitors [9]}

2006 First reports of reduced incidences of CAD as a

2009 First report of reduction of LDL by mAbs.

2012 Phase I trials of mAbs (Alirocumab and Evolocumab) against PCSK9.

US FDA and EMA approval of Alirocumab and

2015 Evolocumab. Approval comes even before completion of Phase II trials.

2016 Plaque regression by Evolocumab. NICE approval of Alirocumab and Evolocumab.

CV outcome trials on Alirocumab and Evolocumab.

\subsection{Shortcomings of $m A b s$}

mAbs target PCSK9 in plasma whereas cholesterol homeostasis is also intracellular.

Frequent subcutaneous injections required (every two weeks / or monthly if given at higher dose).

Expensive treatment limiting their use only in cases of statin unresponsive HeFH \& HoFH.

Possibilities of worsening diabetes mellitus.

PCSK9 binding to LDLr has two phases:

Rapid Phase: $1 / 3^{\text {rd }}$ of overall binding that take place in plasma with half-time of 5-10 minutes and dissociation time of $20 \mathrm{~min}$. mAbs are predominantly active in this domain.

Slow Phase: two third of overall binding with half-time 1.5 hrs and dissociation half-time $>5$ hours. Anti-PCSK9 vaccine address both rapid and slow phase.

Estimating an average blood pool of 5lit in average size human, PCSK9 concentration is $\sim 200 \mathrm{ng} / \mathrm{ml}$. plasma pool turnover is estimated at 2 pools/day. mAbs can address only the plasma pool of PCSK9. Dual inhibition at cellular and plasma level is possible by vaccination. 


\subsection{Development of Anti-PCSK9 Vaccine-siRNA Peptide Vaccine}

Anti-PCSK9 Vaccines using siRNAs are $\sim 20-30$ nucleotide RNA molecules (Inclisiran) is a long-acting synthetic siRNA which is conjugated to triantennary Nacetylgalactosamine carbohydrates. These bind to asialoglycoprotein receptors of hepatocytes leading first to inhibition of intra cellular and then plasma PCSK9 enzymes. A large ambitious global multi-centric Phase $2 \& 3$ Trial (ORION $1-11$ ) is now under way involving approximately 1500 ASCVD patients in North America. ORION-9 trial has enrolled 400 patients with HeFH in North America, Europe, Israel, and South Africa. ORION-5 trial started with 60 patients with $\mathrm{HoFH}$ in Europe, Middle East, and North America [10].

\subsection{Advantage of siRNA Peptide Inclisiran over mAbs}

Extended duration of action: Effects of Inclisiran sodium $300 \mathrm{mgm}$ (equivalent to $284 \mathrm{mg}$ of inclisiran) subcutaneous injections on day 1 , day 90 , and then after 6 months were seen up to 540 days thereafter with primary outcomes in $48 \%$ of the patients. Absolute reduction of LDL-C level by 50 $\mathrm{mg} / \mathrm{dL}$ or $48 \%$ from base level seen at day 180 and continued for 540 days. Furthermore, the two-dose 300-mg Inclisiran regimen caused mean reduction in PCSK9 levels by $69.1 \%$ (p $<0.001$ vs. placebo) and also decreased high sensitivity Creactive protein (hsCRP) by $16.7 \%(\mathrm{p}<0.05)$ [11].

mAbs block plasma PCSK9 whereas Inclisiran vaccine reduces both intra cellular and plasma enzymes to maintain sustained therapeutic effect.

There is absolute reduction in PCSK9 with Inclisiran whereas mAbs cause accumulation of PCSK9 bound to antibody.

\subsection{Unresolved Issue with Inclisiran}

No studies showing the direct effect of inclisiran on intermediate CVD markers, such as intima-media thickness of the carotid artery (CIMT), arterial flow-mediated dilation (FMD), or arterial pulse wave velocity (PWV).

\subsection{Virus-like Particle (VLP)-Peptide Vaccines}

Since 2017, substantial research publications in international journals of repute are coming from Wuhan, China, a place now associated with dubious distinctions. Researches on animal models (Balb/c mice and LDLR mice) with PCSK9Q $\beta-003$ vaccine appears promising [8].

Several other VLP-peptides are under development at different laboratories with potential use in varied chronic diseases and cancer.

Vaccination schedules and doses for VLP-Peptides are under development through human trials.

\subsection{Criteria for Selection of VLP-Peptides as Anti-PCSK9 Vaccine [8]}

Location of peptides: Either on catalytic receptor of
PCSK9 or as regulator of binding between PCSK9 and LDLr. VLP-Peptides match with host immune system.

Ideal antigenicity and hydrophilicity.

Should interact with functional mutation site of PCSK9. CTerminal domain of PCSK9 binds with LDLr.

Amino-acid number should be 10 or less.

Advantage of VLP-Peptides:

VLP-Peptides have been successfully used in many chronic diseases. VLP based anti-angiotensin II receptor vaccine significantly reduced resistant hypertension in animal models [11].

Total Cholesterol (TC) was less by $20 \%$ after $4^{\text {th }}$ vaccination dose. However, no significant change in triglyceride (TG) and High density Lipoprotein (HDL) was observed.

Vaccination worked synergistically with simvastatin $10 \mathrm{mg} / \mathrm{kg} /$ day with $20 \%$ reduction in TC and $60 \%$ reduction of LDL. The effect was observed at 70 days study period.

Free PCSK 9 level diminished by $30 \%$ from baseline at 70 days post vaccination.

Statin therapy inhibit HMG-CoA reductase and increase plasma PCSK 9 by $30 \%$ by a mechanism commonly known as statin paradox. mRNA expression by VLP-peptides increase activity of HMG-CoA reductase in PCSK9Q $\beta-003$ vaccine group and also inhibit PCSK9.

In some studies, vaccination significantly reduced $\mathrm{TC}$, free cholesterol, phospholipids and TG in primates.

\subsection{Safety Issues of Anti-PCSK9 Vaccine}

Based on biological similarity, animal models give us reliable information of efficacy and safety of anti-PCSK9 vaccines for human. $\mathrm{B}$ and $\mathrm{T}$ Cells induce the antibody production in association with $\mathrm{T}$ Helper cells. Effectiveness and safety of vaccines depend on interactions of these with peptide sequence of target PCSK9 enzyme. Keyhole Limpet Homocyanin $(\mathrm{KLH})$ is a rate limiting factor in $\mathrm{T}$ Cell expression against vaccine peptide. In mice models it has been found that despite adequate presence of KLH in T Cells, vaccine peptide did not elicit $\mathrm{T}$ Cell activation after immunization. No abnormal immune mediated histological changes were seen in kidney of vaccinated animals [12].

\subsection{PCSK9 Vaccines in Cancer Treatment}

High LDL and corresponding higher plasma expression of PCSK9 are recently associated with a higher risk of breast cancer [13]. PCSK9 inhibition by vaccination may moderately improve breast cancer outcomes while having no harmful effects in tumour-bearing mice. Similar efficacy was noted in colorectal cancer administered with anti-PCSK9 vaccine in mice model [14].

\section{Conclusion}

Despite unbridled positivism associated with PCSK9 inhibition, there are some conflicting reports which have to be given due consideration. Plasma PCSK9 concentration has 
modest positive relation to $\mathrm{LDL}$, apo B and TG in primary prevention. Strong correlation is lost when all case CVD morbidities and mortalities are taken under consideration in 17 years follow-up [15]. Unlike, baseline apo B, baseline PCSK9 is not a direct predictor of CVD events. On the other hand Laugsand et al [16] in a prospective cohort (1,488 cases vs 3,1819 controls with 11 years follow up) found $47 \%$ higher incidences of MI in highest quartile of PCSK9 in comparison to lowest quartile. However, interestingly, when adjusted to LDL level, the positive association is lost. The progression of coronary artery calcium scores by statin monotherapy was $29.7 \%$, while that by combination therapy with a PCSK9 inhibitor and a statin was $14.3 \%$. Results from secondary prevention are somewhat more encouraging. Higher PCSK9 was associated with severity of CAD in SYNTAX score [17]. A positive association with lower level of plasma PCSK9 was found with less vascular inflammation, lower LDL and positive outcome in ACS in a study involving 2,030 individuals, but failed to predict mortality at one year follow up [18]. This would draw our attention to a landmark study by Ravnskov et al. where authors showed lack of association or an inverse association between LDL and mortality in the elderly over 60 years [19].

Veracity of these findings limit use of anti-PCSK9 treatment within the narrow domain of $\mathrm{HeFH}, \mathrm{HoFH}$ and other non-responders to conventional lipid lowering therapies at comparatively younger age group.

Based on available data, clinical measurement of PCSK9 as a prognostic data or for risk assessment is not recommended. Assuming that LDL-PCSK9 bound moiety is biologically more active form which has failed to produce uniformity in risk prediction, it might be worthwhile to look into pleotropic effect of PCSK9 independent of LDL level.

\section{References}

[1] Warren, JB, et al. Cholesterol Trials and Mortality. Br J Clinical Pharmacol (2016) 82: 168-177.

[2] Ray KK, et al. Statins and all-cause mortality in high-risk primary prevention: a meta-analysis of 11 randomized controlled trials involving 65229 participants. Arch Intern Med 2010; 170: 1024-31.

[3] Nakamura $\mathrm{H}$, et al. Primary prevention of cardiovascular disease with pravastatin in Japan (MEGA Study): a prospective randomised controlled trial. Lancet 2006; 368: 1155-63.

[4] Bulbulia R, et al. Effects on 11-year mortality and morbidity of lowering LDL cholesterol with simvastatin for about 5 years in 20536 highrisk individuals: a randomised controlled trial. Lancet 2011; 378: 2013-20.

[5] Waters DD, et al. (2009) Lipid treatment assessment project 2: a multinational survey to evaluate the proportion of patients achieving low-density lipoprotein cholesterol goals. Circulation 120: 28-34.

[6] Michael D. Shapiro et al. PCSK9: From Basic Science Discoveries to Clinical Trials. Circ Res. 2018 May 11; 122 (10): 1420-1438.

[7] Cameron J, et al. (2006) Effect of mutations in the PCSK9 gene on the cell surface LDL receptors. Hum Mol Genet 15: $1551-1558$.

[8] Yajie Pan et al. A Therapeutic Peptide Vaccine Against PCSK9. Nature.com/scientific reports. 7: 12534 | DOI: 10.1038/s41598-017-13069-w.

[9] Chaudhhary R et al. PCSK9 Inhibitors: A new era of lipid lowering therapy. World J Cardiolo 2017 February 26; 9 (2): 76-91.

[10] Kosmas Constantine E. et al. Inclisiran: A New Promising Agent in the Management of Hypercholesterolemia. Diseases. 2018 Sep; 6 (3): 63.

[11] Chen, X. et al. Efectiveness and Safety of a Terapeutic Vaccine Against Angiotensin II Receptor Type 1 in Hypertensive Animals. HYPERTENSION. 61, 408-416 (2013).

[12] Ryo Kawakami et al. Development of vaccine for dyslipidemia targeted to a proprotein convertase subtilisin/ kexin type 9 (PCSK9) epitope in mice. https://doi.org/10.1371/journal.pone.0191895 February 13, 2018.

[13] Amir Abbas Momtazi-Borojeni, et al. Effects of immunization against PCSK9 in an experimental model of breast cancer. Arch Med Sci 2019; 15 (3): 570-579.

[14] Amir Abbas Momtazi-Borojeni, et al. Potential anti-tumor effect of a nanoliposomal antiPCSK9 vaccine in mice bearing colorectal cancer. Arch Med Sci 2019; 15 (3): 559-559.

[15] Ridker PM, et al. Plasma proprotein convertase subtilisin/kexin type 9 levels and the risk of first cardiovascular events. Eur Heart J. 2016; 37: 554-60. [PubMed: 26508163].

[16] Laugsand LE, et al. Circulating PCSK9 and Risk of Myocardial Infarction. JACC: Basic To Translational Science. 2016; $1: 568-575$.

[17] Li JJ, et al. Proprotein Convertase Subtilisin/Kexin type 9, CReactive Protein, Coronary Severity, and Outcomes in Patients With Stable Coronary Artery Disease: A Prospective Observational Cohort Study. Medicine (Baltimore). 2015; 94: e2426. [PubMed: 26717403].

[18] Gencer B, et al. Prognostic value of PCSK9 levels in patients with acute coronary syndromes. Eur Heart J. 2016; 37: 546-53. [PubMed: 26655339].

[19] Ravnskov U, et al. Lack of an association or an inverse association between low-densitylipoprotein cholesterol and mortality in the elderly: a systematic review. http://dx.doi.org/10.1136/bmjopen-2015-010401. 